\title{
Reflets
}

Revue ontaroise d'intervention sociale et communautaire

\section{Santé mentale et les défis de l'an 2001 : Le paradoxe des choix personnels et professionnels ou l'obsession de l'efficacité}

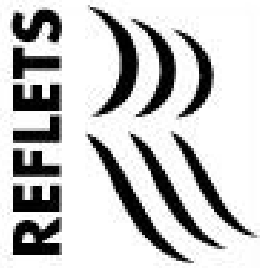

\section{Jean-Marc Bélanger et Michel-André Beauvolsk}

Volume 7, numéro 1, printemps 2001

Santé mentale et les défis de l'an 2001

URI : https://id.erudit.org/iderudit/026333ar

DOI : https://doi.org/10.7202/026333ar

Aller au sommaire du numéro

Éditeur(s)

Reflets : Revue ontaroise d'intervention sociale et communautaire

ISSN

1203-4576 (imprimé)

1712-8498 (numérique)

Découvrir la revue

Citer cet article

Bélanger, J.-M. \& Beauvolsk, M.-A. (2001). Santé mentale et les défis de l'an 2001 : Le paradoxe des choix personnels et professionnels ou l'obsession de l'efficacité. Reflets, 7(1), 10-16. https://doi.org/10.7202/026333ar

Tous droits réservés (C) Reflets : Revue ontaroise d'intervention sociale et communautaire, 2001
Ce document est protégé par la loi sur le droit d'auteur. L'utilisation des services d'Érudit (y compris la reproduction) est assujettie à sa politique d'utilisation que vous pouvez consulter en ligne.

https://apropos.erudit.org/fr/usagers/politique-dutilisation/ 


\section{Santé mentale et les défis de l'an 2001}

\section{Le paradoxe des choix personnels et professionnels ou l'obsession de l'efficacité}

Jean-Marc Bélanger et Michel-André Beauvolsk

Université Laurentienne

Dans son livre Contes à guérir, Contes à grandir, Jacques Salomé présente Le conte du Magasin de la Vie. Ce texte raconte l'histoire d'une petite fille qui visite ce magasin unique au monde. En s'y promenant, elle voit sur un rayon un article appelé le désir de grandir. N'ayant pas d'argent, elle poursuit son exploration. Un peu plus loin, elle aperçoit un étrange flacon. Elle demande à la vendeuse quel en est le prix et celle-ci lui répond qu'on ne peut pas acheter ce flacon, on ne peut que l'échanger. La jeune enfant répond qu'elle ne peut renoncer à ce qu'elle n'a pas reçu.Après plusieurs années, la jeune fille devenue femme revient et ayant réalisé qu'elle possédait maintenant une croyance personnelle, elle décide de la déposer dans les mains de la vendeuse. La vendeuse lui remet alors le flacon du désir de grandir.

Cette métaphore se prête bien au contenu de ce numéro de Reflets sur les défis de l'an 2001 dans le monde de la santé mentale. C'est un récit qui met en prédominance deux grandes valeurs humaines : la capacité de choisir et l'efficacité. La capacité de choisir nous permet de répondre à nos besoins personnels et aussi, au niveau professionnel, de répondre à ceux des autres. Une fois bien équipé personnellement et professionnellement, une fois qu'on a davantage grandi, on peut être efficace. 
Nous avons voulu dans ce numéro explorer les défis de l'an 2001 dans le domaine de la santé mentale en Ontario français. La diversité des champs de pratique et des divers aspects se rapportant à la santé mentale est bien reflétée dans ce numéro en passant par l'épuisement professionnel, le suicide, l'image corporelle, l'ordonnance de traitement, la prostitution, les sans-abri et ainsi de suite. Malgré cette grande diversité, il y a quand même une tendance quant à la livraison des services qui se dessine à travers les instances gouvernementales et dont il faut tenir compte.

Le modèle scientifique se veut la mesure précise d'un phénomène avec le plus grand contrôle de variance possible. Ce processus est linéaire où telle cause produit tel effet. Cette directive en provenance du ministère des Services sociaux et communautaires est très claire et dicte comment traiter les populations cibles. Tout devient mesurable et on peut recenser les effets de ces interventions à l'aide d'outils techniques. C'est l'obsession de l'efficacité qui prend place.

Par contre, dans le domaine du changement social, l'intervenante professionnelle a développé ses habiletés d'aide et d'influence pour rendre le client le plus autonome possible dans ses décisions et dans ses choix. Ce processus est circulaire où telle cause peut produire plusieurs effets très différents. C'est ce qu'on a toujours considéré être l'aide véritable.

Lorsque l'obsession de l'efficacité entre en jeu, l'intervenante n'est plus aidante mais voit alors son rôle redéfini en technicienne devant démontrer l'efficacité de ses interventions en utilisant différentes techniques de mesure. Sa formation est confrontée à cette nouvelle exigence pour laquelle elle n'est pas très bien préparée.

Traditionnellement l'intervenante a toujours interagi avec ses clientes et clients qui partagent certaines dimensions communes et qui présentent une variance de comportements, de réactions et d'émotions, ce qui est contraire aux phénomènes physiques mesurés par les scientifiques. On est alors confronté à deux défis majeurs : la redéfinition extérieure du rôle de l'intervenante, et la mesure de l'intervention. 
Redéfinir le rôle de quelqu'un de l'extérieur consiste à nier sa valeur professionnelle et ses fonctions. En d'autres mots, c'est lui enlever ses choix. C'est aussi nier ses habiletés pour mieux servir l'illusion de l'efficacité. Il faut alors se demander par rapport à quoi on mesure l'efficacité? Par rapport au problème présenté? Par rapport au contexte social? Par rapport aux coûts? Par rapport à la durée des services?

Cette efficacité ne fait que régler un problème de structure dans une société où le système social est en besoin. Pour lui permettre de fonctionner et lui donner plus longue vie, on doit lui donner son souffle et, pour ce faire, on donne préséance à ce système et non à l'individu. C'est efficace en termes de résultats, mais où aboutira l'individu en bout de ligne?

Pour ce qui est de la mesure de l'intervention, que prétendon mesurer? L'être humain, même s'il dit vouloir changer, ne le fait que très lentement. Lui fait-on encore cette place ou la lui at-on enlevée?

Comment répondre aux contrastes qui se manifestent dans l'organisation de la vie? Les contrastes font référence aux attributs des individus et des groupes qui marquent leur identité tels la classe sociale, le sexe, l'ethnie, l'orientation sexuelle et l'incapacité, pour n'en nommer que quelques-uns. Les problèmes de santé mentale ont besoin d'être considérés et conciliés pour que l'adaptation sociale puisse se réaliser. L'inclusion est la notion de fournir l'égalité d'opportunité et éventuellement l'égalité des résultats pour les groupes non dominants dans la société.

Les impératifs de répondre aux éléments de diversité sont doubles en intervention sociale. Il faut d'abord faire avancer et faire respecter les valeurs de la profession qui réferent spécifiquement au besoin pour l'équité, puis accorder la priorité à la justice sociale qui doit de plus en plus entrer dans l'arène de la responsabilité sociale.

Comme intervenantes et intervenants sociaux, il faut s'éloigner de la croyance que nous n'avons rien à donner. Nous avons beaucoup à offrir à notre clientèle, et ce, malgré les diverses contraintes systémiques et politiques. Le conte du Magasin de la Vie 
nous confronte à faire nos choix. Les choix sont quasi illimités et tous contiennent autant d'avantages que de désavantages. D'un point de vue professionnel, nous pouvons alors poser la question à savoir si ce choix nous rend plus efficace.

Ce numéro de Reflets nous présente une variété de textes sur différentes thématiques de la santé mentale. L'entrevue réalisée auprès de Michelle Bernier-Wilson met en évidence la situation contextuelle des besoins en santé mentale en Ontario, surtout au niveau des services aux enfants. Elle retient deux dossiers prioritaires du gouvernement, soit les services mobiles d'intervention d'urgence et la télépsychiatrie, tout en soulignant que le réseau des Centres de santé mentale pour enfants n'est qu'un petit élément de tous les services consacrés à l'enfance. Par la suite, elle dépeint le mandat particulier du Centre de l'enfant et de la famille, en décrivant la clientèle servie, le genre de services disponibles et les défis auxquels l'agence fait face. Elle termine l'entrevue en discutant des particularités pour les services de santé mentale à l'intention des populations francophone et amérindienne.

Dans la rubrique Dossier nous vous présentons quatre articles de fond sur des thèmes fort variés, mais aussi d'actualité. L'article de Julie Gagnon sur la loi 68 en l'honneur de Brian Smith fait état des ordonnances de traitement communautaire obligatoire pour les personnes psychiatrisées. Elle nous présente le concept derrière cette initiative ainsi que son contexte, tout en soulevant les enjeux qui en ressortent. L'auteure souligne à la fin de son article certaines interventions alternatives. Le deuxième article traite des sans-abri, un phénomène beaucoup plus présent et visible dans de nombreuses communautés de tous les coins de l'Ontario. Carol Kauppi, Jean-Marc Bélanger et Jennifer Keck nous font connaitre la perspective des fournisseurs de services dans diverses agences communautaires dans la région de Sudbury. On nous rappelle encore une fois que certains problèmes sociaux (pauvreté, chômage, logements à prix abordable) sont au coeur même du phénomène des sans-abri, et que ce n'est pas uniquement relié à des problèmes de santé mentale. Plusieurs sans-abri avec des problèmes de santé mentale pourraient mieux fonctionner dans leur communauté s'il y avait suffisamment de ressources disponibles 
pour contrer le problème de pauvreté. Anselme Mvilongo et Michel-André Beauvolsk nous font part d'un problème omniprésent en santé mentale qu'on retrouve malheureusement trop souvent chez les jeunes : le suicide. Les auteurs explorent les facteurs de vulnérabilité qui mènent à l'acte suicidaire et soulignent le lien qui existe entre le suicide et la santé mentale. Cette rubrique se termine avec une critique du programme Express, qui est un programme de L'Ontario au travail. Mary Ann Jenkins, Adje van de Sande et Jean-Marc Bélanger présentent leur critique à partir d'une perspective socialiste féministe. Les auteurs démontrent les limites reliées à un programme obligatoire qui se donne comme objectif de rompre le cycle vicieux de la dépendance sur l'aide sociale. En particulier, les auteurs font mention de diverses problématiques telles la pauvreté, la dépression maternelle, la violence, l'allaitement, le rôle parental et le soin de l'enfant, lesquels sont des thèmes reliés de près ou de loin à la santé mentale des jeunes mères bénéficiaires du programme.

Dans la rubrique Des pratiques à notre image, cinq articles font la tournée de divers sujets captivants. Michel-André Beauvolsk et Jean-Marc Bélanger donnent un compte-rendu d'entrevues effectuées auprès d'un groupe de cliniciennes et de cliniciens en santé mentale du Centre de l'enfant et de la famille de Sudbury. Basées sur les éléments théoriques concernant les résultats de la thérapie, ces entrevues ont eu pour but de permettre à l'équipe clinique d'exposer la réalité de leurs interventions. On peut ainsi bénéficier de l'expérience d'une équipe d'une agence pour mieux saisir les enjeux auxquels elle fait face. SophieVinette a rédigé un superbe article sur l'image corporelle des femmes. Plus de $90 \%$ des femmes, soutient-elle, se trouvent trop grosses. L'auteure explore les causes de cette insatisfaction, qui a des conséquences sur l'image de soi et, par ricochet, sur la santé mentale. Comme troisième article, Michel-André Beauvolsk fait le bilan d'une intervention auprès d'une adolescente dépressive en milieu scolaire. S'inspirant du modèle symbolique expérienciel de Carl Whitaker, l'auteur nous démontre comment il a utilisé les symboles, la musique et le jeu pour faciliter la prise de conscience et appuyer le changement voulu par la cliente. Par la suite, Le combat de 
Catherine exemplifie les contradictions et les contraintes auxquelles fait face une cliente en services de santé mentale pour avoir accès à son dossier. En raison de la nature délicate du sujet et pour sauvegarder la confidentialité des parties en cause, ce texte est anonyme. Enfin, la rubrique Des pratiques à notre image se termine avec un article sur l'épuisement professionnel (burnout). Viviane Munroe et Nicole Brunette ont mené une étude auprès d'intervenantes et d'intervenants francophones du nord-est de l'Ontario sur l'épuisement professionnel et sur le stress dans le but d'évaluer les niveaux de stress ressentis par ces derniers. Ce sujet est fort pertinent pour toute personne faisant de l'intervention dans les domaines sociaux, de santé et de santé mentale.

Dans la rubrique Aux quatre coins de la province, Marie-Hélène Gérome nous fait part d'une recherche effectuée par le Réseau national d'action éducation femmes (RNAÉF) sur les comportements de santé en lien avec l'image corporelle des adolescentes francophones. En plus de présenter un aperçu des comportements de santé des adolescentes vivant en contexte francophone minoritaire, l'objectif de la recherche sera d'identifier des revendications et des pistes d'action en faveur de la promotion de la santé pour ce groupe particulier. C'est définitivement un sujet d'actualité et une recherche à suivre. Cette rubrique présente également un témoignage de Julie Joanisse sur Le profil de Philippe. Julie a voulu partager son expérience personnelle et familiale par rapport à son vécu comme tierce personne, témoin de services de santé mentale promulgués à son frère. Sombre sort, maintenant qu'il est adulte, il y a plus de services à sa disponibilité que lorsqu'il était adolescent.

Finalement, nous aimerions attirer votre attention sur l'article de Marie Josée Jobin sur la prostitution. L'auteure est récipiendaire de la bourse RIFAS 2000 de l'École de service social de l'Université d'Ottawa. Elle a voulu comprendre par sa recherche ce qu'est la prostitution féminine à partir du discours des femmes qui la vivent. Cette étude est centrée sur la théorie de l'étiquetage dans le champ d'activité de la prostitution et a pour but de mieux comprendre comment les femmes qui font de la prostitution se perçoivent et aussi comment elles perçoivent leur environnement. 
Nous croyons que ce numéro couvre plusieurs dimensions du domaine de la santé mentale. Nous avons fait appel aux sources disponibles et possibles et sommes heureux du résultat. Notre expérience personnelle ressemble à celle de la petite fille dans $L e$ conte du Magasin de la Vie; nous avons aussi grandi. Nous espérons que la lecture de ces textes vous invitera à faire des choix qui vous permettront aussi de grandir. Bonne lecture!

\section{Bibliographie}

Salomé, Jacques (1993), Contes à guérir, Contes à grandir, Paris : Albin Michel, pages 311-315. 